\title{
INVARIANT SUBSPACES FOR PRODUCTS OF HERMITIAN OPERATORS
}

\author{
HEYDAR RADJAVI AND PETER ROSENTHAL
}

\begin{abstract}
It is shown that a nonscalar operator which is the product of a Hermitian operator and a positive operator has a nontrivial hyperinvariant subspace; this is a slight generalization of a result of Suzuki's.
\end{abstract}

Suzuki [3] has shown that an operator $T$ which is the product of two positive operators has a nontrivial hyperinvariant subspace; (i.e., a closed linear manifold, different from $\{0\}$ and the entire space, which is left invariant by every operator which commutes with $T$ ). We present a simple proof of a slight generalization of Suzuki's result.

THEOREM. If $T$ is the product of a positive operator and a Hermitian operator, (and $T$ is not a multiple of the identity), then $T$ has a nontrivial hyperinvariant subspace.

Proof. Assume that $T=R K$ where $R$ is positive and $K$ is Hermitian; (if the product occurs in the other order simply apply the following to $T^{*}$ ). If $R$ or $K$ has a nontrivial nullspace then so does $T$ or $T^{*}$, and the nullspace of an operator is hyperinvariant. Assume that $R$ and $K$ are injective; they then also have dense ranges since they are Hermitian. Now $R$ has a unique positive square root $R^{1 / 2}$. It is trivial to verify the fact that

$$
T R^{1 / 2}=R^{1 / 2}\left(R^{1 / 2} K R^{1 / 2}\right)
$$

and

$$
\left(R^{1 / 2} K\right) T=\left(R^{1 / 2} K R^{1 / 2}\right)\left(R^{1 / 2} K\right) .
$$

Since $R^{1 / 2} K$ and $R^{1 / 2}$ are injective and have dense ranges, the operator $T$ is quasi-similar to the Hermitian operator $R^{1 / 2} K R^{1 / 2}$ and has a nontrivial hyperinvariant subspace by the well-known result of Sz. Nagy-Foias (cf. [2], [1, p. 103]).

It appears to be much more difficult to prove that the product of two Hermitian operators has an invariant subspace; if the above were extended

Received by the editors November 10, 1972.

AMS (MOS) subject classifications (1970). Primary 47A15.

Key words and phrases. Operators on Hilbert space, hyperinvariant subspace, positive, Hermitian.

(c) American Mathematical Society 1974 
to the product of a positive operator and a unitary operator then, by the polar decomposition, the invariant subspace problem would be solved.

\section{REFERENCES}

1. P. A. Fillmore, Notes on operator theory, Van Nostrand Reinhold Math. Studies, no. 30, Van Nostrand-Reinhold, New York, 1970. MR 41 \#2414.

2. B. Sz.-Nagy and C. Foiaş, Analyse harmonique des opérateurs de l'espace de Hilbert, Masson, Paris; Akad. Kiadó, Budapest, 1967; English rev. transl., NorthHolland, Amsterdam; American Elsevier, New York; Akad. Kiadó, Budapest, 1970. MR 37 \#778; 43 \#947.

3. N. Suzuki, Reduction theory of operators on Hilbert space, Indiana Univ. Math. J. 20 (1971), 953-958.

Department of Mathematics, University of Toronto, Toronto, Canada (Current address of Peter Rosenthal)

Current address (Heydar Radjavi): Department of Mathematics, Dalhousie University, Halifax, Nova Scotia, Canada 